\title{
Triptolide exerts an anti-tumor effect on non-small cell lung cancer cells by inhibiting activation of the IL-6/STAT3 axis
}

\author{
YING HUANG, ZHE CHEN, YU WANG, XIN BA, YAO HUANG, PAN SHEN, HUI WANG and SHENGHAO TU \\ Institute of Integrated Traditional Chinese and Western Medicine, Tongji Hospital, Tongji Medical College, \\ Huazhong University of Science and Technology, Wuhan, Hubei 430030, P.R. China
}

Received November 7, 2018; Accepted April 23, 2019

DOI: $10.3892 /$ ijmm.2019.4197

\begin{abstract}
Lung cancer is the leading cause of cancer-associated mortality and current treatments are not sufficiently effective. Numerous studies have revealed that triptolide (TP), a classical traditional Chinese medicine compound widely used as an anti-inflammatory and antirheumatic drug, also has an antitumor effect. This effect is hypothesized to be mediated by multiple pathways, with signal transducer and activator of transcription 3 (STAT3) possibly one of them. Evidence indicates that STAT3 participates in the initiation and progression of lung cancer during cell proliferation, apoptosis and migration; however, whether and how TP affects STAT3 and its targets remain unclear. In this study, the potential role of TP in the proliferation, apoptosis, and migration of non-small cell lung cancer cell lines was investigated and evaluated the impact of TP on the interleukin-6 (IL-6)/STAT3 axis. The results showed that TP inhibited cell proliferation and migration and induced apoptosis. TP decreased the phosphorylation of STAT3, inhibited STAT3 translocation into the nucleus, and reduced the expression of STAT3 target genes involved in cell survival, apoptosis and migration, e.g. $C-m y c, B C L-2$, myeloid cell leukemia-1 (MCL-1), and matrix metallopeptidase 9 (MMP-9). Additionally, IL-6-induced activation of STAT3 target genes (e.g. $M C L-1$ and $B C L-2$ ) was attenuated by TP and homoharringtonine. In conclusion, the effect of TP on STAT3 signaling points to a promising strategy for drug development.
\end{abstract}

\section{Introduction}

Lung cancer is the leading cause of cancer-related deaths worldwide, imposing a heavy social and economic burden in

Correspondence to: Dr Shenghao Tu, Institute of Integrated Traditional Chinese and Western Medicine, Tongji Hospital, Tongji Medical College, Huazhong University of Science and Technology, Jiefang Avenue, 1095 Qiaokou Street, Wuhan, Hubei 430030, P.R. China

E-mail: shtu@tjh.tjmu.edu.cn

Key words: lung cancer, triptolide, signal transducer and activator of transcription 3, chemotherapy
China (1). Non-small cell lung cancer (NSCLC) accounts for $\sim 85 \%$ of lung cancer histological subtypes (2). The majority of NSCLC cases are diagnosed at an advance stage $(\sim 70 \%)$, with patients missing the chance for radical surgery and thus facing chemotherapy as the main treatment option (3). Despite the excitement after the shift from empirical therapy to more effective and better tolerated regimens for NSCLC over the past two decades, the 5-year overall survival rate remains as low as $4-17 \%$ depending on the stage and regional differences (4). What makes the situation worse is that among patients with NSCLC, after a transient response to initial treatment, drug resistance of malignant cells occurs frequently. Therefore, finding an effective and safe anti-tumour agent is one of the highest-priority goals in lung cancer research.

Signal transducer and activator of transcription 3 (STAT3) is an important member of the STAT family and is recognised as a key oncogenic factor highly relevant to NSCLC (5). STAT3 plays crucial roles in cancer initiation and progression by binding to the promoter regions of a number of genes that encode regulators of cell proliferation (such as cyclin DI and $M Y C$ ), survival (such as BCL-2 and myeloid cell leukemia-1 (MCL-1)], inflammation [such as interleukin-6 (IL-6) and cyclooxygenase-2], and metastasis [such as matrix metallopeptidase 9 (MMP-9) and vimentin] (6-8). STAT3 activation is transient and dynamic under physiological conditions. However, activated STAT3 is persistently present in $50 \%$ of NSCLC tissue (9) due to stimulation of an upstream signaling pathway (10), suggesting that STAT3 is a lung cancer-specific target for anticancer therapy. Activation of cell cycle and survival genes enables STAT3-expressing cells to escape damage from conventional chemotherapeutic drugs (11); thus, the STAT3 oncogenic pathway is involved in drug resistance (12). Similarly, a meta-analysis has revealed that (13) expression of STAT3 or phosphorylated STAT3 (p-STAT3) is a strong predictor of poor prognosis among patients with NSCLC (14). Hence, interference with the STAT3 pathway might counteract the resistance to chemotherapy and exert an adjuvant effect during the treatment of lung cancer.

Because current standard treatments are far from ideal for patients with NSCLC, a natural compound with multiple advantages has come to our attention (15). Triptolide (TP), a classical natural compound, has been demonstrated to exert anti-tumour effects on ovarian cancer (16), breast cancer (17), hepatocellular carcinoma (18) and lung cancer (19). Studies have revealed that low-dose TP helps to circumvent resistance, 
enhances effectiveness and relieves adverse effects of anticancer therapies $(20,21)$. It has been reported that TP exerts its anti-tumour activities in terms of cell proliferation, cell death, angiogenesis, and metastasis (22). Nevertheless, at present, the potential influence of TP on IL-6/STAT3 signalling in NSCLC is still unknown.

In this study, we determined the effect of TP on the proliferation, apoptosis, and invasiveness of NSCLC cells and investigated the potential underlying mechanisms by evaluating low dose TP's influence on the IL-6/STAT axis.

\section{Materials and methods}

Materials. TP ( $>98 \%$ purity) was purchased from Dalian Meilun Co., Ltd. Human recombinant IL-6 (cat. no. 200-06; Peprotech, Inc.) was resolved and stored according to the manufacturer's instructions. 3-(4,5-Dimethylthiazol-2-yl)-2, 5-diphenyltetrazolium bromide (MTT) and protease inhibitor cocktail (cat. no. 04693132001) were purchased from Sigma-Aldrich (Merck KGaA). Antibodies against anti-cleaved caspase-3 (cat. no. GB11009) were purchased from Servicebio. Antibodies against BCL-2 (cat. no. 182858) and MMP-9 (cat. no. 76003) were purchased from Abcam, MCL-1 (cat. no. 94296), cyclin D1 (cat. no. 2978S), C-myc (cat. no. 5605S), STAT3 (cat. no. 4904) and p-STAT3 (cat. no. 9145) were acquired from Cell Signaling Technology Inc., and antibodies against $\beta$-actin (cat. no. BB0710) were from Biossci Biotechnology Co., Ltd. DAPI, Triton X-100 and an anti-fade mounting medium were purchased from Servicebio. The Annexin V/PI Apoptosis Detection kit (cat. no. KGA105-KGA108) was from Nanjing KeyGen Biotech Co., Ltd. Propidium iodide (PI) and the JC-1 Staining kit were acquired from Beyotime Institute of Biotechnology. DyLight 488-conjugated goat anti-rabbit IgG antibody (cat. no. A24221) was from Abbkine Scientific Co., Ltd.

Cell culture. Human lung adenocarcinoma PC9 cells sensitive to epidermal growth factor receptor (EGFR) with low expression of IL-6 and human alveolar basal epithelial adenocarcinoma A549 cells with higher expression of IL-6 were provided by the Caner Laboratory, Tongji Medical College (Wuhan, China). The cells were cultured in RPMI-1640 medium (HyClone; GE Healthcare Life Sciences) supplemented with 10\% FBS (Gibco; Thermo Fisher Scientific, Inc.), $100 \mathrm{U} / \mathrm{ml}$ penicillin, and $100 \mu \mathrm{g} / \mathrm{ml}$ streptomycin at $37^{\circ} \mathrm{C}$ in a humidified incubator with $5 \% \mathrm{CO}_{2}$ and $95 \%$ air.

MTT assay. Cells were seeded in 96-well plates at a density of $5 \times 10^{3}$ cells per well and cultured overnight. A series of concentrations ranging to $500 \mathrm{nM}$ TP were added into the medium. After either 24 or $48 \mathrm{~h}$, MTT was added to assay cell viability. Absorbance at $570 \mathrm{~nm}$ was recorded on a microplate reader (Synergy 2; BioTek Instruments, Inc.). The inhibition ratio was calculated as 1 -optical density $(\mathrm{OD})_{\text {treated }} / \mathrm{OD}_{\text {control. }}$ The $\mathrm{IC}_{50}$ values were calculated by plotting the drug concentration versus the percent cell viability of the cells after TP intervention for 24 and $48 \mathrm{~h}$.

Flow cytometry. At $3 \times 10^{5}$ cells per well were exposed to TP in 12 wells for $24 \mathrm{~h}$ after overnight serum starvation. Thereafter, the cells were washed with PBS and fixed with $70 \%$ ethanol at $4^{\circ} \mathrm{C}$ overnight. Then, cell cycle analyses were performed through flow cytometry according to the manufacturer's instructions (Beyotime Institute of Biotechnology). Apoptosis was analyzed via pre-treatment with a selected concentration of TP for $24 \mathrm{~h}$ (PC9 cells) or $48 \mathrm{~h}$ (A549 cells) and then was detected with the Annexin V/PI Staining kit (Nanjing KeyGen Biotech Co., Ltd.) and assayed by flow cytometry (BD FACS Calibur; BD Biosciences). Cells undergoing early-stage apoptosis were categorized as Annexin $\mathrm{V}^{+} / \mathrm{PI}^{-}$, whereas Annexin $\mathrm{V}^{+} / \mathrm{PI}^{+}$cells were assumed to be at a late stage of apoptosis. Subsequently, mitochondrial trans-membrane potential $\Delta \Psi$ ) was determined according to the manufacturer's instructions using the JC-1 Apoptosis Detection kit (Beyotime Institute of Biotechnology). Data analysis was conducted using the FlowJo software (oxs 10.6; FlowJo LLC).

Transwell assay. Transwell migration assays were performed using 24-well Transwell inserts ( $8 \mu \mathrm{m}$ pore size; Costar; Corning, Inc.), as described previously (23). A total of $1 \times 10^{5}$ cells were pre-treated with TP for $24 \mathrm{~h}$, then harvested and resuspended in RPMI-1640 (HyClone; GE Healthcare Life Sciences) at a concentration of $10^{5}$ cells $/ \mathrm{ml}$. Next, $200 \mu \mathrm{l}$ of the cell suspension mixed with $750 \mu 1$ RPMI-1640 medium supplemented with $10 \%$ FBS was added to the upper chamber, and incubated for another $24 \mathrm{~h}$ at $37^{\circ} \mathrm{C}$. Non-migratory cells were gently wiped from the top surface of the membrane with a cotton swab. Migratory cells on the bottom side of the membrane were rinsed with PBS, fixed in $4 \%$ paraformaldehyde and stained with $0.1 \%(\mathrm{~m} / \mathrm{v})$ crystal violet for $10 \mathrm{~min}$ at room temperature. Images were captured under a ZEISS digital microscope (magnification x100; Carl Zeiss AG). The migration rate was quantified by counting the stained cells in selected 3-5 fields of view.

Western blotting. Cells were treated with or without TP for $24 \mathrm{~h}$, then cells were washed twice with ice-cold PBS and lysed in RIPA buffer containing a protease inhibitor cocktail (Thermo Fisher Scientific, Inc.). Soluble proteins were collected after centrifugation at $12,000 \mathrm{x} \mathrm{g}$ for $15 \mathrm{~min}$ at $4^{\circ} \mathrm{C}$. Protein concentration was determined by the bicinchoninic acid (BCA) method. Subsequently, $50 \mu \mathrm{g}$ of each protein sample was separated by SDS-PAGE on a $10-12 \%$ gel $(120 \mathrm{~V}, 90 \mathrm{~min})$ and transferred to a PVDF membrane (Bio-Rad Laboratories, Inc.) at $280 \mathrm{~mA}$ for $90 \mathrm{~min}$. After blocking with 5\% non-fat milk for $1 \mathrm{~h}$ at room temperature, the membranes were incubated with specific primary antibodies $(1: 1,000)$ at $4^{\circ} \mathrm{C}$ overnight. After three washes with TBS containing $0.1 \%$ Tween 20 , the membranes were incubated with fluorescently-labeled secondary antibodies $(1: 20,000)$ for $1 \mathrm{~h}$ at room temperature. The bands were visualized using a near-infrared fluorescence imaging system (Odyssey; LI-COR Biosciences). Band densities were quantified in the ImageJ software (version 1.52 National Institutes of Health). In addition, cells were pretreated with TP or homoharringtonine (HHT) for $24 \mathrm{~h}$, then $50 \mathrm{nM}$ IL-6 (cat. no. 200-06; PeproTech, Inc.) was added for another $30 \mathrm{~min}$, finally cells were harvested and lysed for western blotting.

Immunofluorescence. Cells $\left(10^{5}\right.$ cells/well) were seeded on glass cover slides. After treatment with $60 \mathrm{nM}$ TP or $4 \mu \mathrm{M}$ 
A
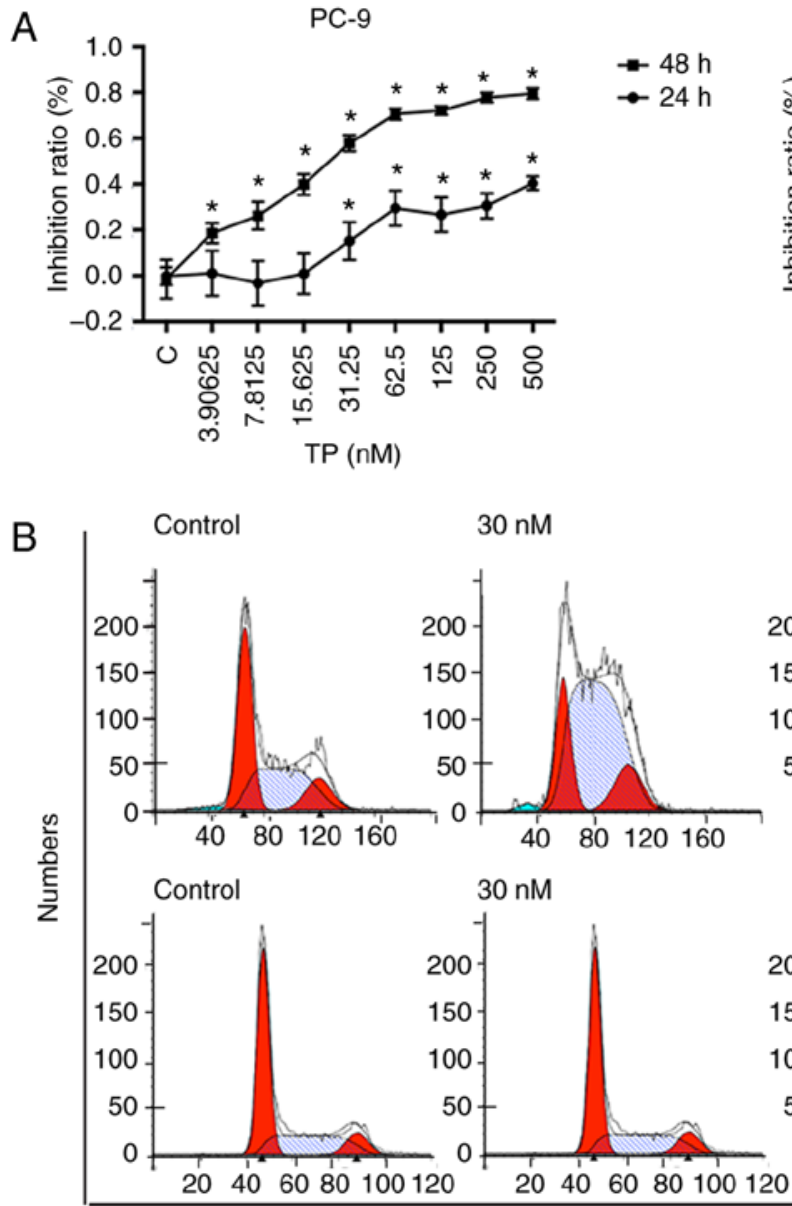
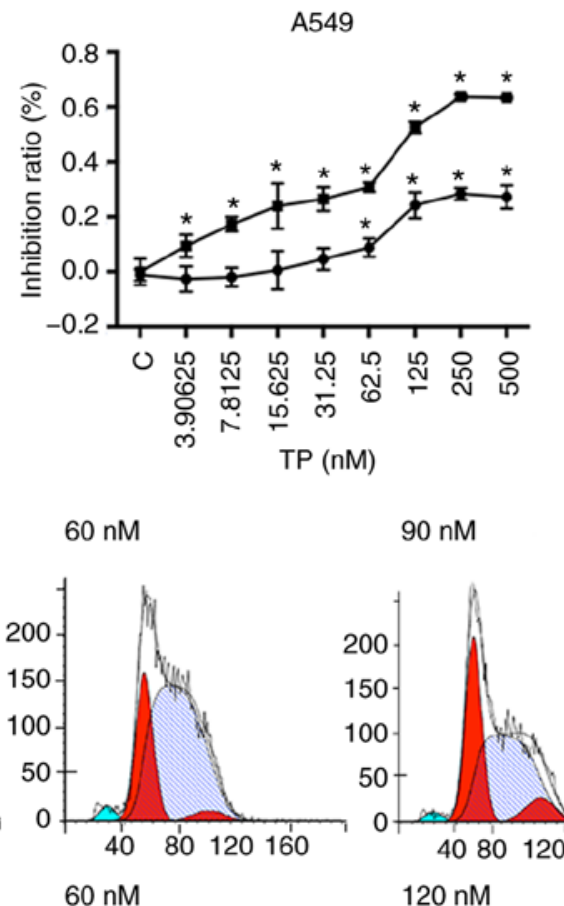

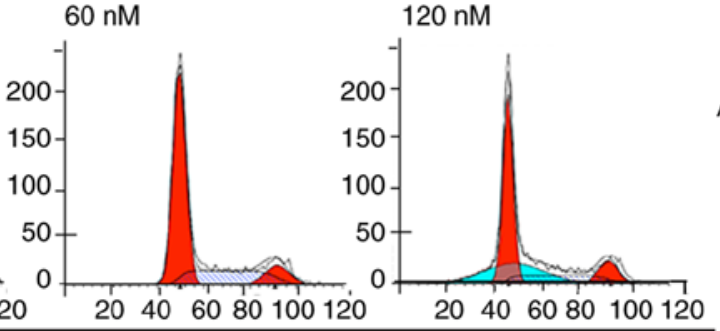

Channels (PI-A)
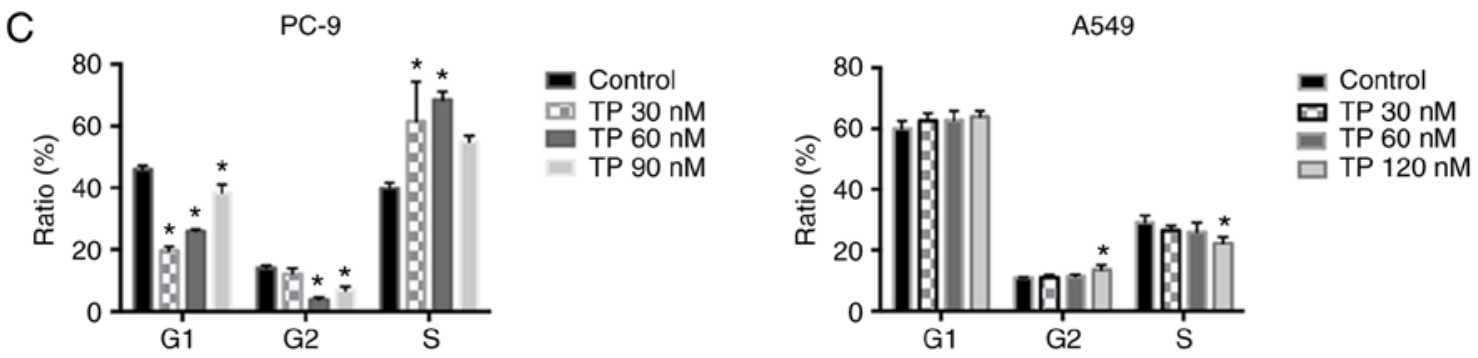

Figure 1. TP impairs cell viability by inducing cell cycle arrest in non-small cell lung cancer cells. (A) PC9 and A549 were treated with series concentrations for 24 or $48 \mathrm{~h}$, and then detected by MTT assay. (B) Cell cycle profiles obtained by flow cytometry (PI DNA staining) in PC9 and A549 cells. (C) Triptolide influenced cell cycle arrest in the G-S phase in PC9 and slightly increased S phase in A549. "P<0.05 vs. control. TP, triptolide; PI, propidium iodide.

STAT3 inhibitor for $24 \mathrm{~h}$, the cells were fixed with $4 \%$ buffered formalin for $30 \mathrm{~min}$ at room temperature, permeabilized with $0.5 \%$ Triton X-100, and blocked with $10 \%$ goat serum in PBS (Servicebio) for $30 \mathrm{~min}$ at room temperature. After successive incubation with the primary antibody $(1: 100)$ at $4^{\circ} \mathrm{C}$ for $12 \mathrm{~h}$, a secondary antibody (DyLight 488-conjugated goat anti-rabbit IgG; $1: 8,000$ ) was incubated for another $1 \mathrm{~h}$ at room temperature, at last cells were stained with DAPI (1:500) in the dark for $5 \mathrm{~min}$. The fluorescence signals were captured using a fluorescence microscope (Nikon Eclipse CI; Nikon Corporation).

Statistical analysis. All experiments were conducted in triplicate and repeated three times. Representative results are shown as the mean $\pm \mathrm{SD}$ and were plotted using Prism 6.0 (GraphPad
Software, Inc.). Statistical calculations were performed via one-way ANOVA followed by the Student-Newman-Keuls test. $\mathrm{P}<0.05$ was considered to indicate a statistically significant difference.

\section{Results}

TP impairs viability of NSCLC cells by inducing cell cycle arrest. To evaluate the cytotoxic action of TP, PC9 and A549 cells were treated with a series of TP concentrations ranging from 0 to $500 \mathrm{nM}$ for either 24 or $48 \mathrm{~h}$. The $\mathrm{IC}_{50}$ was calculated using $\log$ formula. The $\mathrm{IC}_{50}$ of PC9 and was $1,238 \pm 243.8 \mathrm{nM}$ and the $\mathrm{IC}_{50}$ of $\mathrm{A} 549$ was $1,339 \pm 150.2 \mathrm{nM}$. After $48 \mathrm{~h}$ of treatment with TP, the corresponding values were $29.33 \pm 2.102$ and $135.9 \pm 0.9328$ nM. As shown in Fig. 1A, TP was cytotoxic 

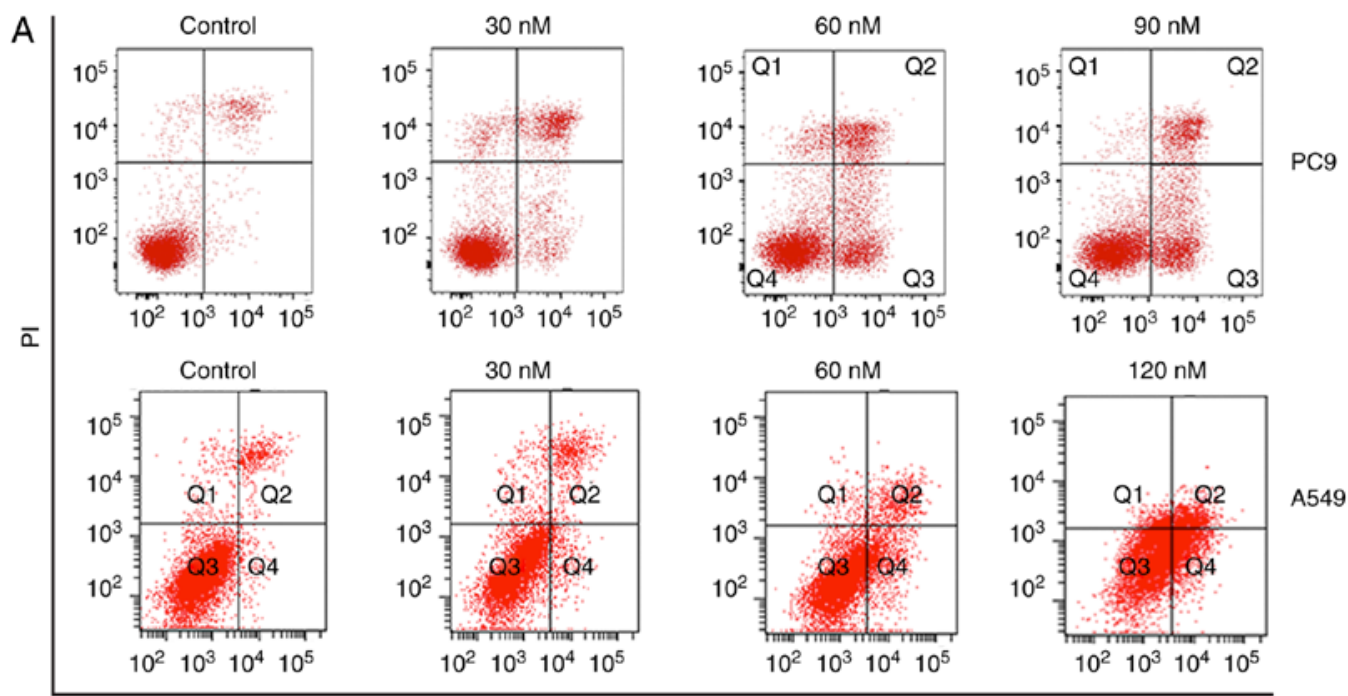

Annexin V-FITC
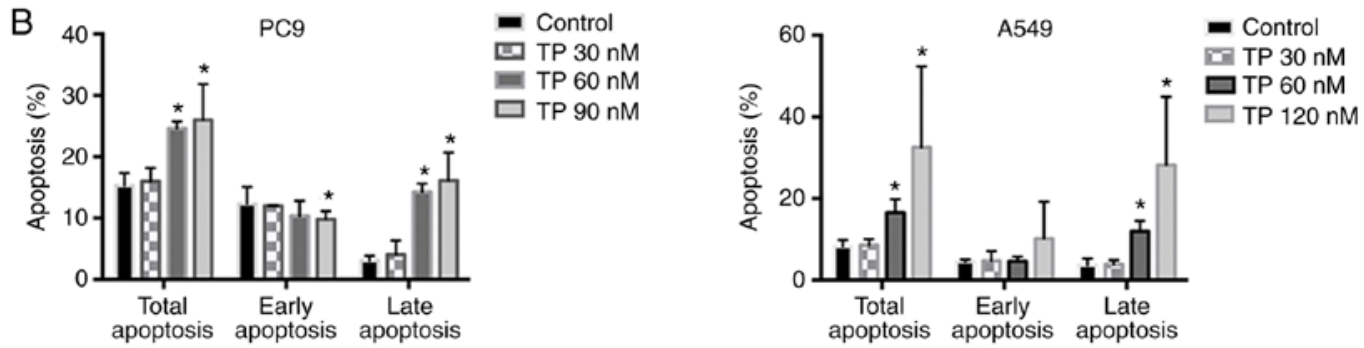

C
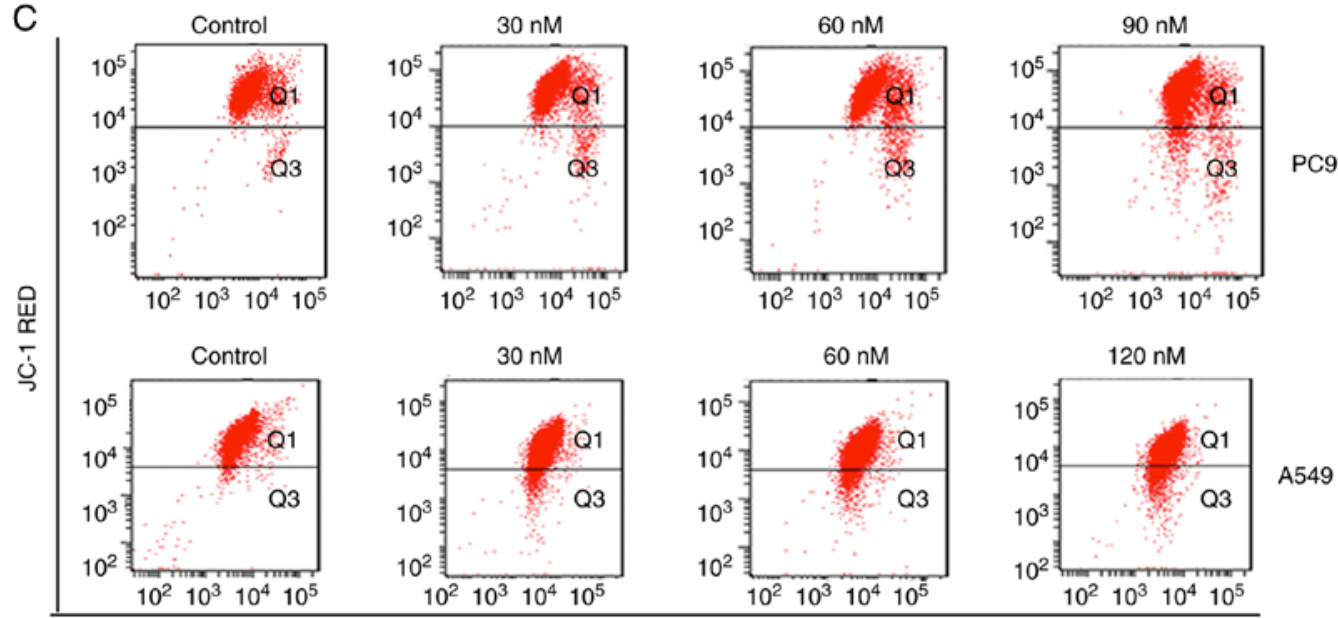

JC-1 BLUE
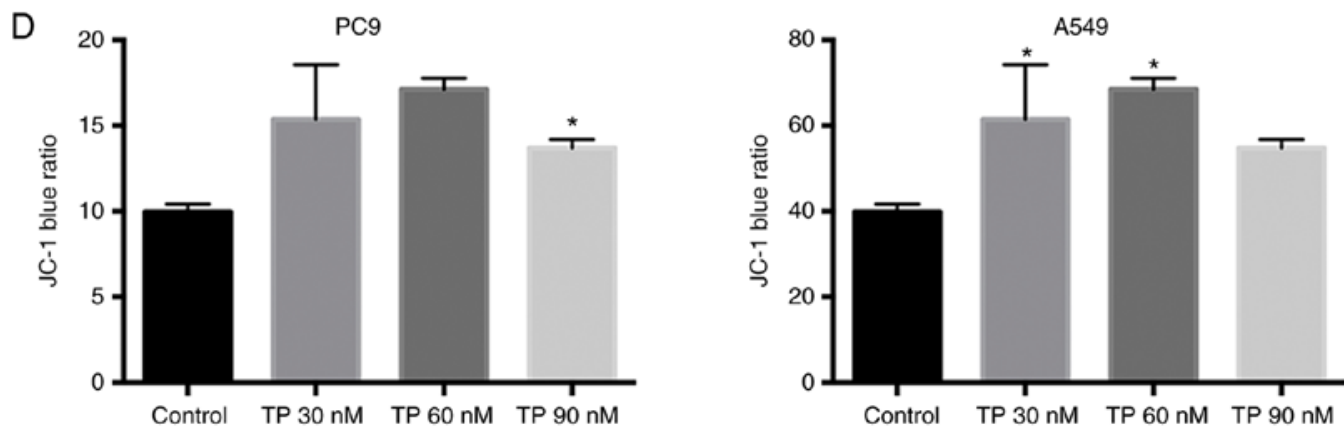

Figure 2. TP disrupts active mitochondria and induces apoptosis in non-small cell lung cancer cells. (A) PC9 were treated with TP at selected concentration 30 , 60 and $90 \mathrm{nM}$ or without TP (control) for $24 \mathrm{~h}$, and A549 were treated with 30, 60 and $120 \mathrm{nM}$ for $48 \mathrm{~h}$, and cells were harvested and apoptosis was detected. Early apoptotic cells were Annexin $\mathrm{V}^{+} / \mathrm{PI}^{-}$, and late apoptotic cells were Annexin $\mathrm{V}^{+} / \mathrm{PI}^{+}$. (B) The number of apoptotic cells (second quadrant and fourth quadrant which showed Annexin $\mathrm{V}^{+}$) were significantly increased after TP treatment compared with the control group. (C and D) Mitochondrial membrane potential assay was performed using JC-1. When the cell membrane potential is lowered, a transition from red fluorescence to blue fluorescence is detected. The cells under the horizontal gate were indicate reduced mitochondrial membrane potential. Statistical results are expressed as the mean $\pm \mathrm{SD}$. ${ }^{*} \mathrm{P}<0.05 \mathrm{vs}$. control. TP, triptolide; PI, propidium iodide. 


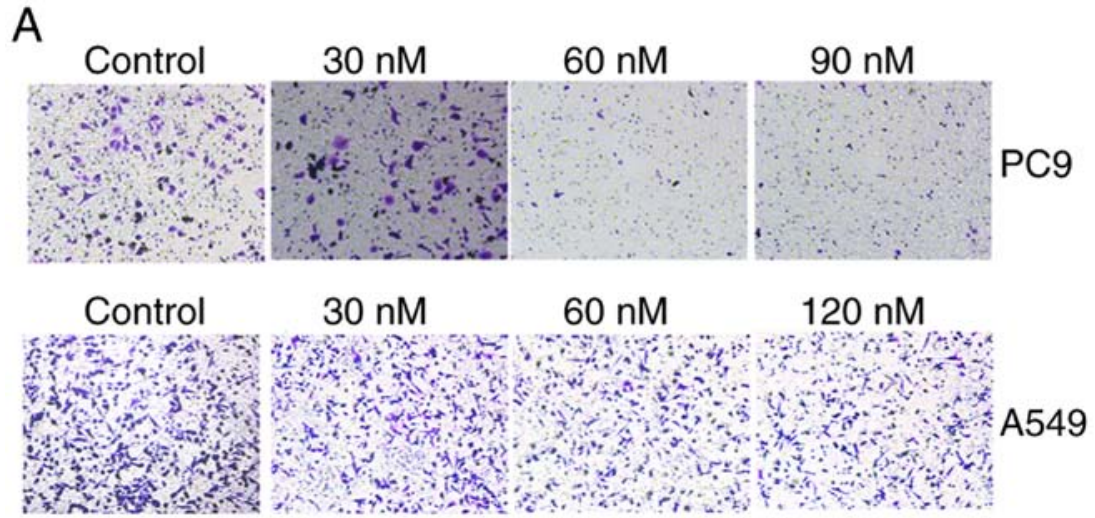

B
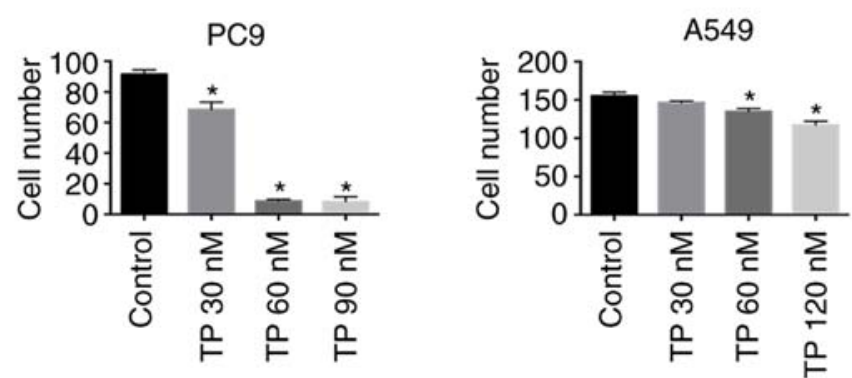

Figure 3. TP inhibited migration of non-small cell lung cancer cells. (A) The in vitro chamber migration assay was used to detect the effects of TP on cell migration in PC9 and A549. (B) Quantification of PC9 and A549 cell migration following TP treatment. " $\mathrm{P}<0.05$ vs. control, TP, triptolide.

after $48 \mathrm{~h}$ treatment even at a low concentrations $(\mathrm{P}<0.05)$. $\mathrm{TP}$ inhibited proliferation (according to an inhibition ratio) of PC9 and A549 cells at $48 \mathrm{~h}$ in a dose-dependent manner. During the $24 \mathrm{~h}$ treatment, TP at concentrations $<15.625 \mathrm{nM}$ was not cytotoxic at all toward PC9 and A549 cells. The inhibition of proliferation started to be noticeable via a sharp slope in the inhibition ratio (15.625-62.500 nM in PC9 cells and 15.625-125.000 nM in A549 cells) (24) followed by a relatively flat plateau at high TP concentrations. Therefore, these comparatively low doses were selected to conduct subsequent experiments.

Whether TP influences cell viability by affecting the cell cycle was examined (Fig. 1B). On the basis of the inhibition ratio, two concentrations within the sharp-slope region (30 and $60 \mathrm{nM})$ and one concentration in the plateau region $(90 \mathrm{nM}$, PC-9; 120 nM, A549) were selected. As shown in Fig. 1C, TP at the concentration in the sharp-slope region $(30$ and $60 \mathrm{nM})$ strongly promoted the G1/S arrest in PC9 cells. There was a slight increase in the number of cells in the $S$ phase at $90 \mathrm{nM}$ TP as well, but the change was not statistically significant. Similar results were not seen in corresponding experiments on A549 cells after treatment with TP for $24 \mathrm{~h}$. A slight decrease in the number of cells in the $S$ phase at $120 \mathrm{nM}$ TP was observed, though it appeared that TP in general did not affect the cell cycle in A549 cells (Fig. 1C).

TP disrupts active mitochondria and induces apoptosis in NSCLC cells. It was subsequently analyzed whether TP treatment has any impact on apoptosis. Starting from the concentration of $60 \mathrm{nM}$, treatment of PC9 cells with TP for $24 \mathrm{~h}$ caused a significant increase in total apoptosis
(Fig. 2A and B). In contrast, after $48 \mathrm{~h}$ treatment of A549 cells with TP, total apoptosis increased strongly, and this change was attributed to a late-apoptosis population (Fig. 2A and B). Then, $\Delta \Psi$ was measured as disruption of mitochondria is regarded as a distinctive feature of the intrinsic pathway of apoptosis. As demonstrated by the JC-1 blue ratio, treatment with TP for $24 \mathrm{~h}$ slightly decreased $\Delta \Psi$ (Fig. 2C and D). These results suggested that TP induced apoptosis at least partially at an early stage by disrupting active mitochondria.

TP inhibits migration of NSCLC cells. Considering the antimetastasis effect of TP, we an in vitro chamber migration assay was performed to test whether TP affects NSCLC cell migration. As presented in Fig. 3, the number of migratory PC9 cells was decreased by TP treatment and dropped dramatically at 60 and $90 \mathrm{nM}$; whereas the number of migratory A549 cells stayed largely unchanged upon TP treatment; however, a statistically significant decrease in the number of migratory cells was observed after treatment with 60 and $120 \mathrm{nM}$ TP, but this was less dramatic than the effect in PC9 cells.

TP disturbs an activated STAT3 pathway in NSCLC cells. Aberrant and constitutive activation of STAT3 in NSCLC has been reported (9) and is associated with tumor progression and a poor prognosis (14). After treatment with TP for $24 \mathrm{~h}$, p-STAT3 was decreased in PC9 (Fig. 4A) and A549 cells (Fig. 4B). The mRNA expression of PC9 (Fig. S1) and A549 (Fig. S2) was consistent with protein expression. As shown in Fig. S3, the ratio of p-STAT3/total STAT3 was marginally decreased by TP in A549 cells, with statistical significance at $120 \mathrm{nM}(\mathrm{P}<0.05)$. 
A

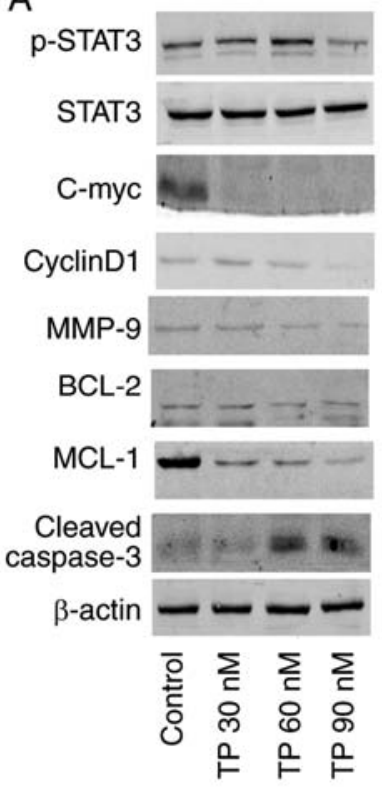

B

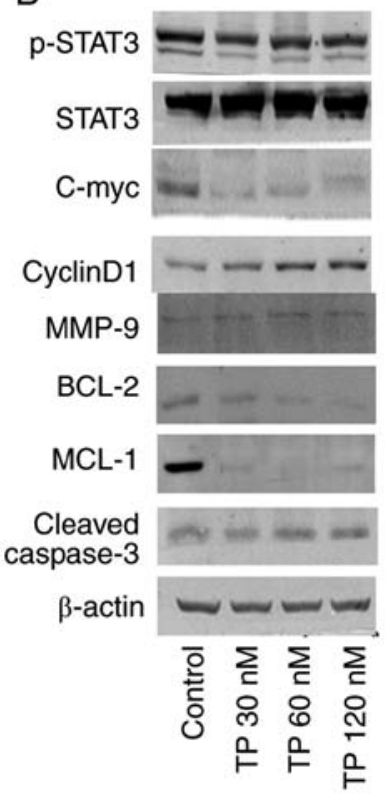

Figure 4. TP disturbs activated STAT3 pathway in non-small cell lung cancer cells. Total and phosphorylation of STAT3 were detected in (A) PC9 and (B) A549. Proteins downstream of STAT3 involved in cell proliferation (C-myc, cyclin D1), protein involved in apoptosis (MCL-1, BCL-2 and cleaved caspase-3) and migration (MMP-9) was detected in p-, phosphorylated; STAT3, signal transducer and activator of transcription 3; TP, triptolide; MMP-9, matrix metallopeptidase 9; MCL-1, myeloid cell leukemia-1.

Expression levels of target genes of STAT3 were also examined. C-myc expression was found to greatly decreased upon TP treatment in PC9 (Fig. 4A) and A549 cells (Fig. 4B). Although cyclin D1 was downregulated by TP in PC9 cells (Fig. 4B), cyclin D1 was upregulated by TP treatment in A549 cells (Fig. 4B). The overexpression of cyclin D1 may contribute to resistance to TP (25), as well as cell DNA damage (24). Caspase- 3 is a critical executioner of apoptosis, as it is either partially or totally responsible for the proteolytic cleavage of many key proteins, and cleaved caspase- 3 is the active form of the protein. As shown in PC9 cells (Fig. 4A), TP increased the level of cleaved caspase-3. Expression of anti-apoptotic proteins MCL-1 and BCL-2 was decreased after TP treatment in a dose-dependent manner in PC9 (Fig. 4A) and A549 cells (Fig. 4B). The two bands of BCL-2 seen in Fig. 4A may be homedimers or poor antibody specificity (26). TP also downregulated MMP-9 expression in a dose-dependent manner in PC9 (Fig. 4A) and A549 cells (Fig. 4B). The above results suggested that TP treatment inhibited cell proliferation, induced apoptosis and inhibited cell migration by suppressing the activation of STAT3.

Signal transduction in the STAT3 pathway, as visualized by STAT3 translocation into the nucleus, was inhibited by TP treatment. Less prevalent and weaker STAT3 staining was observed in cell nuclei in the TP treatment groups (Fig. 5).

TP attenuated STAT3 activation induced by IL-6. Elevated IL-6 expression has been observed in many patients with lung cancer (27). A549 cells appear to be much less sensitive to TP compared with PC9 cells, thus it was speculated that the expression of IL- 6 in these cells is associated with the

results. The effect of TP in the presence of exogenous IL-6 in PC9 cells was analyzed, with a non-specific IL-6/STAT3 inhibitor HHT serving as a positive control, which prevents the initial elongation step of protein synthesis (Fig. 6). Simlar to Ham et al (28), IL-6 seems not significantly increase the phosphorylation of STAT3, but did impair the ability of HHT to inhibit STAT3 phosphorylation. IL-6 appeared to induce expression of the STAT3 downstream target gene, BCL-2, but there was no statistical difference $(\mathrm{P}>0.05)$. At the same time, TP and HHT could reduce the expression of BCL-2 compared with IL-6-stimulated cells. Compared with the control group, the expression of MCL-1 was significantly increased by exogenous IL-6 $(\mathrm{P}<0.05)$. Similar to HHT, pretreated TP can almost completely block MCL-1 expression. Briefly, TP might enhance the effects of chemotherapy by inhibiting IL-6-induced MCL-1 activation.

\section{Discussion}

Even though treatment of NSCLC has changed dramatically over the past two decades, only modest improvements have been achieved in terms of the patient survival rate (4). Drug resistance and side effects are still obstacles to successful cancer therapy. With the advantages of low toxicity and cost, natural compounds have been regarded as a promising adjuvant therapies for NSCLC (29). Initially prescribed as an anti-inflammatory drug, TP is considered an alternative therapeutic agent for the treatment of a wide range of cancers, such as pancreatic cancer and colon cancer (30), ovarian cancer (31), breast cancer (32), hepatocellular carcinoma (33) and lung cancer (34). A distinct antitumour activity of TP lies in its antagonism of drug resistance $(20,31)$. This beneficial effect might be mediated by changes in ATP-binding cassette transporters (35), induction of apoptosis pathways, increase in tumor suppressors and decrease in oncogenic factors, and interactions with the RNA polymerase II complex (36) by TP. Aside from mitogen-activated protein kinase (32) and Wnt/ $\beta$-catenin pathways $(33)$, the IL-6/JAK/STAT3 axis $(34,37)$ is also also involved in the antitumor activity of TP in cancer types other than lung cancer. However, at present, the influence of TP on IL-6/STAT3 signaling during the treatment of NSCLC is still unclear.

Although the antitumor effect of TP has been described previously, the current study further confirms how low doses of TP exert anticancer effects or adjuvant to chemotherapy in lung cancer through IL-6/STAT3. In this study, the antitumor effects of TP on PC9 and A549 were analyzed. After balancing effectiveness and possible toxicity, appropriate doses were selected to conduct subsequent experiments. TP inhibited cell proliferation, promoted cell apoptosis and cell migration at a comparative dose in PC9 cells, while TP decreased the expression of genes associated apoptosis resistance, such as MCL-1. Studies indicated that MCL-1 is a key target of adjuvant chemotherapy to reverse the cisplatin-resistance in NSCLC (38). The direct antitumor effect in PC9 and the adjuvant effect of circumventing resistance in A549 make it superior to other traditional Chinese therapeutic agents. TP exerted antiproliferative action on both NSCLC cell lines. In agreement with other findings (39), the data showed significant S phase cell cycle arrest in PC9 cells. Such S phase arrest 
A
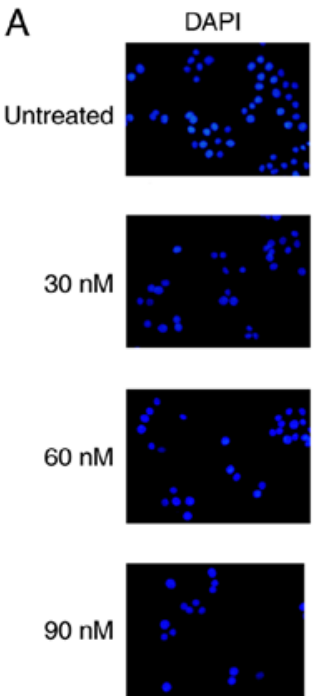

STAT3
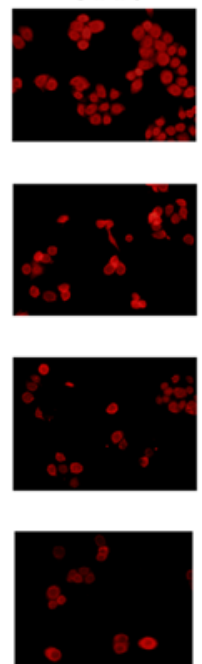
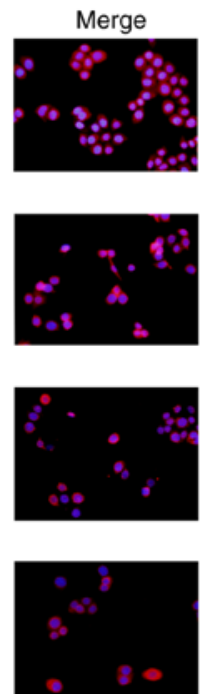

B
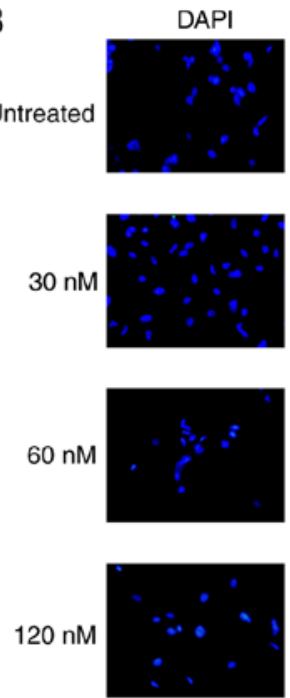
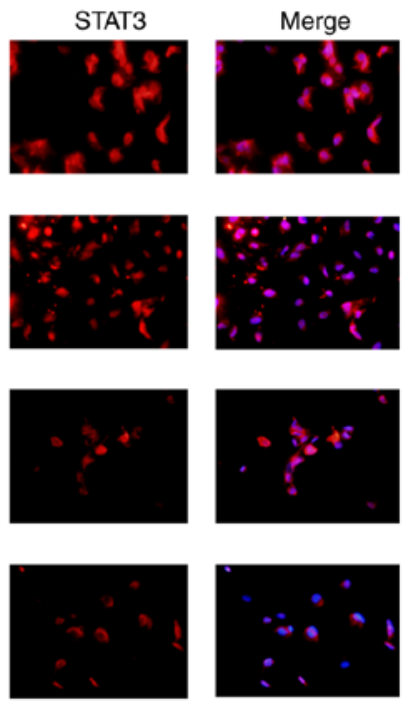

Figure 5. Phosphorylation of STAT3 was detected by immunofluorescence. Cells were treated with or without TP, the nuclear translocation was detected by immunofluorescence, (A) PC9 and (B) A549 was either untreated or treated with different concentrations of TP. Cells were stained with anti-phosphotyrosine 705-STAT3 antibody (red) and counterstained with DAPI (blue), decreased after treatment with different concentrations of TP compared with control. STAT3, signal transducer and activator of transcription 3; TP, triptolide.

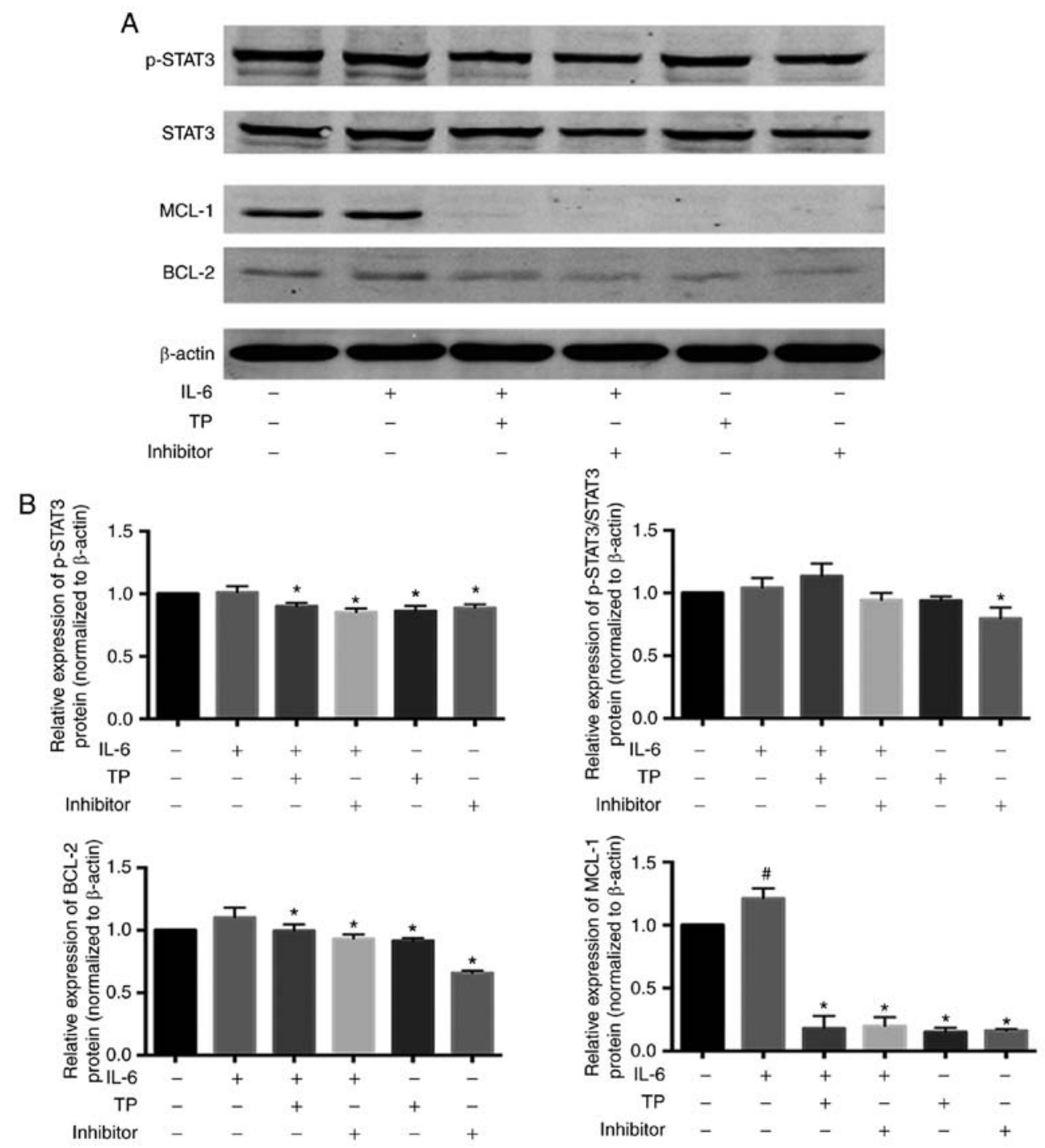

Figure 6. TP decreased activation of STAT3 induced by IL-6. (A) Protein expression of p-STAT3, P-STAT3/STAT3, BCL-2, and MCL-1 were detected by western blot analysis. (B) Image $\mathrm{J}$ was used to analyze the intensity of the band. ${ }^{\prime \prime} \mathrm{P}<0.05$ vs. untreated; ${ }^{*} \mathrm{P}<0.05$ vs. exogenous IL- 6 treatment. p-, phosphorylated; STAT3, signal transducer and activator of transcription 3; MCL-1, myeloid cell leukemia-1; IL-6, interleukin-6; TP, triptolide. 
was not significant in A549 cells after treatment with TP for $24 \mathrm{~h}$. Unexpectedly, there was a decrease of DNA synthesis in the cells treated with $120 \mathrm{nM}$ TP; DNA synthesis change might be a consequence of increased apoptosis observed in the same experimental setting. In any case, the weaker effect of TP on cell cycle arrest in A549 cells compared with PC9 cells is worthy of further investigation. C-myc and cyclin D1 are crucial factors that are associated with STAT3 activation and cell proliferation. A decrease in both $\mathrm{C}$-myc expression and cyclin D1 expression was seen in PC9 cells treated with $\mathrm{TP}$; this result echoes the aforementioned findings and further elucidates the TP-induced cell cycle arrest in $\mathrm{S}$ phase. In parallel, C-myc was also found to be downregulated by TP in A549 cells, whereas TP elevated the cyclin D1 level in A549 cells. Overexpression cyclin D1 may cause resistance to cytotoxic drugs (40), thus limiting the effects of TP. However, high levels of cyclin D1 prime cells for an enhanced DNA damage response (24), causing sustained cyclin D1-cyclin-dependent kinase activity, leading to inappropriate replication of DNA and chromosomal damage $(41,42)$. This result may be the reason why $\mathrm{S}$ phase arrest was barely detectable among TP-treated A549 cells. On the basis of the literature $(24,43)$, it is speculated that TP enhances DNA damage and arrests the cell cycle in the $S$ phase to inhibit cell proliferation.

Bypassing apoptosis is a critical step in carcinogenesis. The apoptosis pathway, as a result, has been a hot target for many anticancer therapies. As many as $22-65 \%$ of NSCLC cells aberrantly express apoptotic proteins; this feature malignantly enhances cell proliferation and promotes resistance to chemotherapy. In this study, TP markedly increased the percentage of cells undergoing early apoptosis in PC9 and A549 cells. Activated caspase-3 is a typical hallmark of apoptosis (44). The findings of the present study showed that TP increases the expression of cleaved caspase-3 in NSCLC cells. Other authors $(13,45)$ have also stated that inhibition of STATs directly decreases the levels of anti-apoptotic proteins, such as BCL-2 and MCL-1, which are known to facilitate tumor cell survival. The results of the current study revealed that TP treatment decreased the expression of BCL-2 and MCL-1. Given that TP reduced the phosphorylation of STAT3 in PC9 and A549 cells, it was concluded that TP induces apoptosis of NSCLC cells through the STAT3/MCL-1 axis.

Aberrant activation of STAT3 contributes to cancer progression in human tumors (46). It induces MMP-9, which has an important role in cell invasion and metastasis (47). Activated MMP-9 degrades collagens in the extracellular matrix, and this activity is closely associated with the invasive and metastatic potential of numerous types of solid tumor (48-50). The findings of the current study clearly indicate that TP downregulates MMP-9 expression and inhibits NSCLC cell migration, thus indicating that TP inhibits the STAT3/MMP-9 axis.

The present study demonstrated that TP inhibited cell proliferation and migration, and induced apoptosis by suppressing STAT3 activation and downregulating targets of STAT3. The finding that TP inhibited STAT3 phosphorylation and its nuclear translocation is consistent with data from studies on colitis-related colon cancer (37), multiple myeloma cells (51), and pancreatic cancer (52). The results of the current study show that A549 cells are less responsive to TP, which may be associated with the expression of IL-6 in these cells. NSCLC cells carry mutations in oncogenes $\operatorname{Eg} f r(45 \%)$ and Kras (25\%) (13); these mutations drive the overexpression of IL-6. Indeed, IL-6 is overexpressed in many patients with lung cancer (27). Hyperactivation of IL-6 could activate STAT3, which in turn produces more IL-6 $(5,13,53)$. In the present study, the influence of TP on STAT3 activation by exogenous IL-6 was analyzed. IL-6 significantly upregulated MCL-1 expression in PC9 cells, and MCL-1 has been demonstrated to be associated with chemoresistance in oral squamous cell carcinoma (54), so IL-6 might be a factor involved in drug resistance. Additionally, IL-6 significantly reduced the ability of HHT to inhibit STAT3 phosphorylation, that is possible reason why A549 showed smaller response to TP than PC9. These data are indicative of a promising application of TP in lung cancer management.

Although appropriate methods have been used to illustrate the hypothesis, it is difficult to demonstrate the exact mechanism of TP against NSCLC. The effect of TP on downstream target genes of STAT3 indicated the possible existence of another regulator, and more research is needed to confirm this conclusion. This preliminary study illustrated that TP has promising effects on NSCLC, a clinical investigation using combined TP with conventional therapy is necessary determine the effects in recurrent advanced NSCLC. In conclusion, the IL-6/STAT3 pathway appears to be another therapeutic target in NSCLC, and TP may have antitumor effects or at least an adjuvant effect.

\section{Acknowledgements}

The authors acknowledge the gift of cells from Tongji Cancer Laboratory.

\section{Funding}

This study was supported by the National Natural Science Foundation of China (grant no. 81573802).

\section{Availability of data and materials}

The datasets used and/or analyzed during the current study are available from the corresponding author on reasonable request.

\section{Authors' contributions}

YiH, ZC and ST conceived and designed the study. YiH, YW, $\mathrm{XB}$ and $\mathrm{YaH}$ performed the experiments. $\mathrm{YiH}$ and ST wrote the paper. YiH, ZC and ST reviewed and edited the manuscript. PS and HW provided assistance for data acquirement, statistical analysis and manuscript editing. All authors read and approved the manuscript.

\section{Ethics approval and consent to participate}

Not applicable.

\section{Patient consent for publication}

Not applicable. 


\section{Competing interests}

The authors declare that they have no competing interests.

\section{References}

1. Hong QY, Wu GM, Qian GS, Hu CP, Zhou JY, Chen LA, Li WM, Li SY, Wang K, Wang Q, et al: Prevention and management of lung cancer in China. Cancer 121 (Suppl 17): S3080-S3088, 2015.

2. Herbst RS, Morgensztern D and Boshoff C: The biology and management of non-small cell lung cancer. Nature 553: 446-454, 2018.

3. Shi Y, Sun Y, Ding C, Wang Z, Wang C, Wang Z, Bai C, Bai C, Feng J, Liu X, et al: China experts consensus on icotinib for non-small cell lung cancer treatment (2015 version). J Thorac Dis 7: E468-E472, 2015.

4. Hirsch FR, Scagliotti GV, Mulshine JL, Kwon R, Curran WJ Jr, Wu YL and Paz-Ares L: Lung cancer: Current therapies and new targeted treatments. Lancet 389: 299-311, 2017.

5. Gao SP, Mark KG, Leslie K, Pao W, Motoi N, Gerald WL, Travis WD, Bornmann W, Veach D, Clarkson B and Bromberg JF: Mutations in the EGFR kinase domain mediate STAT3 activation via IL-6 production in human lung adenocarcinomas. J Clin Invest 117: 3846-3856, 2007.

6. Yu H, Pardoll D and Jove R: STATs in cancer inflammation and immunity: A leading role for STAT3. Nat Rev Cancer 9: 798-809, 2009.

7. Bournazou E and Bromberg J: Targeting the tumor microenvironment: JAK-STAT3 signaling. JAKSTAT 2: e23828, 2013.

8. Bromberg JF, Wrzeszczynska MH, Devgan G, Zhao Y, Pestell RG, Albanese C and Darnell JE Jr: Stat3 as an oncogene. Cell 98: 295-303, 1999.

9. Li CJ, Li YC, Zhang DR and Pan JH: Signal transducers and activators of transcription 3 function in lung cancer. J Cancer Res Ther 9 (Suppl 2): S67-S73, 2013.

10. Grivennikov SI and Karin M: Dangerous liaisons: STAT3 and NF-kappa B collaboration and crosstalk in cancer. Cytokine Growth Factor Rev 21: 11-19, 2010.

11. Brown JM and Wilson G: Apoptosis genes and resistance to cancer therapy: What does the experimental and clinical data tell us? Cancer Biol Ther 2: 477-790, 2003.

12. Barré B, Vigneron A, Perkins N, Roninson IB, Gamelin E and Coqueret O: The STAT3 oncogene as a predictive marker of drug resistance. Trends Mol Med 13: 4-11, 2007.

13. Yan X, Li P, Zhan Y, Qi M, Liu J, An Z, Yang W, Xiao H, Wu H, Qi Y and Shao H: Dihydroartemisinin suppresses STAT3 signaling and Mcl-1 and Survivin expression to potentiate ABT-263-induced apoptosis in non-small cell lung cancer cells harboring EGFR or RAS mutation. Biochem Pharmacol 150: 72-85, 2018.

14. Xu YH and Lu S: A meta-analysis of STAT3 and phospho-STAT3 expression and survival of patients with non-small-cell lung cancer. Eur J Surg Oncol 40: 311-317, 2014.

15. Mishra BB and Tiwari VK: Natural products: An evolving role in future drug discovery. Eur J Med Chem 46: 4769-4807, 2011.

16. Liu L, Xiong X, Shen M, Ru D, Gao P, Zhang X, Huang C, Sun Y, $\mathrm{Li} \mathrm{H}$ and Duan Y: Co-delivery of triptolide and curcumin for ovarian cancer targeting therapy via mPEG-DPPE/CaP nanoparticle. J Biomed Nanotechnol 14: 1761-1772, 2018.

17. Klauber-DeMore N, Schulte BA and Wang GY: Targeting MYC for triple-negative breast cancer treatment. Oncoscience 5 : 120-121, 2018

18. Li SG, Shi QW, Yuan LY, Qin LP, Wang Y, Miao YQ, Chen Z, Ling CQ and Qin WX: C-Myc-dependent repression of two oncogenic miRNA clusters contributes to triptolide-induced cell death in hepatocellular carcinoma cells. J Exp Clin Cancer Res 37: 51, 2018.

19. Mao X, Tong J, Wang Y, Zhu Z, Yin Y and Wang Y: Triptolide exhibits antitumor effects by reversing hypermethylation of WIF1 in lung cancer cells. Mol Med Rep 18: 3041-3049, 2018.

20. Hou ZY, Tong XP, Peng YB, Zhang BK and Yan M: Broad targeting of triptolide to resistance and sensitization for cancer therapy. Biomed Pharmacother 104: 771-780, 2018.

21. Beglyarova N, Banina E, Zhou Y, Mukhamadeeva R, Andrianov G, Bobrov E, Lysenko E, Skobeleva N, Gabitova L, Restifo D, et al: Screening of conditionally reprogrammed patient-derived carcinoma cells identifies ERCC3-MYC interactions as a target in pancreatic cancer. Clin Cancer Res 22: 6153-6163, 2016
22. Meng C, Zhu H, Song H, Wang Z, Huang G, Li D, Ma Z, Ma J, Qin Q, Sun X and Ma J: Targets and molecular mechanisms of triptolide in cancer therapy. Chin J Cancer Res 26: 622-626, 2014

23. Chang L, Lei X, Qin YU, Zhang X, Jin H, Wang C, Wang X, $\mathrm{Li} \mathrm{G}$, Tan C and Su J: MicroRNA-133b inhibits cell migration and invasion by targeting matrix metalloproteinase 14 in glioblastoma. Oncol Lett 10: 2781-2786, 2015.

24. Li Z, Jiao X, Wang C, Shirley LA, Elsaleh H, Dahl O, Wang M, Soutoglou E, Knudsen ES and Pestell RG: Alternative cyclin D1 splice forms differentially regulate the DNA damage response. Cancer Res 70: 8802-8811, 2010.

25. Lamb J, Ramaswamy S, Ford HL, Contreras B, Martinez RV, Kittrell FS, Zahnow CA, Patterson N, Golub TR and Ewen ME: A mechanism of cyclin D1 action encoded in the patterns of gene expression in human cancer. Cell 114: 323-334, 2003.

26. Dremina ES, Sharov VS and Schöneich C: Heat-shock proteins attenuate SERCA inactivation by the anti-apoptotic protein Bcl-2: Possible implications for the ER $\mathrm{Ca}^{2+}$-mediated apoptosis. Biochem J 444: 127-139, 2012.

27. Duan S, Tsai Y, Keng P, Chen Y, Lee SO and Chen Y: IL-6 signaling contributes to cisplatin resistance in non-small cell lung cancer via the up-regulation of anti-apoptotic and DNA repair associated molecules. Oncotarget 6: 27651-27660, 2015.

28. Ham IH, Oh HJ, Jin H, Bae CA, Jeon SM, Choi KS, Son SY, Han SU, Brekken RA, Lee D and Hur H: Targeting interleukin-6 as a strategy to overcome stroma-induced resistance to chemotherapy in gastric cancer. Mol Cancer 18: 68, 2019.

29. Sharma SB and Gupta R: Drug development from natural resource: A systematic approach. Mini Rev Med Chem 15: 52-57, 2015.

30. Yan $P$ and Sun $X$ : Triptolide: A new star for treating human malignancies. J Cancer Res Ther 14 (Suppl): S271-S275, 2018.

31. Jiang N, Dong XP, Zhang SL, You QY, Jiang XT and Zhao XG: Triptolide reverses the Taxol resistance of lung adenocarcinoma by inhibiting the $\mathrm{NF}-\kappa \mathrm{B}$ signaling pathway and the expression of NF- $\kappa$ B-regulated drug-resistant genes. Mol Med Rep 13: 153-159, 2016.

32. Teng F, Xu Z, Chen J, Zheng G, Zheng G, Lv H, Wang Y, Wang L and Cheng X: DUSP1 induces apatinib resistance by activating the MAPK pathway in gastric cancer. Oncol Rep 40: 1203-1222, 2018.

33. Li X, Lu Q, Xie W, Wang Y and Wang G: Anti-tumor effects of triptolide on angiogenesis and cell apoptosis in osteosarcoma cells by inducing autophagy via repressing Wnt $/ \beta$-catenin signaling. Biochem Biophys Res Commun 496: 443-449, 2018.

34. Fan D, He X, Bian Y, Guo Q, Zheng K, Zhao Y, Lu C, Liu B, $\mathrm{Xu} X$, Zhang $\mathrm{G}$ and Lu A: Triptolide modulates TREM-1 signal pathway to inhibit the inflammatory response in rheumatoid arthritis. Int J Mol Sci 17: 498, 2016.

35. Chen J, Gao J, Yang J, Zhang Y and Wang L: Effect of triptolide on the regulation of ATP-binding cassette transporter A1 expression in lipopolysaccharide-induced acute lung injury of rats. Mol Med Rep 10: 3015-3020, 2014.

36. Vispé S, DeVries L, Créancier L, Besse J, Bréand S, Hobson DJ, Svejstrup JQ, Annereau JP, Cussac D, Dumontet C, et al: Triptolide is an inhibitor of RNA polymerase I and II-dependent transcription leading predominantly to down-regulation of short-lived mRNA. Mol Cancer Ther 8: 2780-2790, 2009.

37. Wang Z, Jin H, Xu R, Mei Q and Fan D: Triptolide downregulates Racl and the JAK/STAT3 pathway and inhibits colitis-related colon cancer progression. Exp Mol Med 41: 717-727, 2009.

38. Ma J, Zhao Z, Wu K, Xu Z and Liu K: MCL-1 is the key target of adjuvant chemotherapy to reverse the cisplatin-resistance in NSCLC. Gene 587: 147-154, 2016.

39. Oliveira A, Beyer G, Chugh R, Skube SJ, Majumder K, Banerjee S, Sangwan V, Li L, Dawra R, Subramanian S, et al: Triptolide abrogates growth of colon cancer and induces cell cycle arrest by inhibiting transcriptional activation of E2F. Lab Invest 95: 648-659, 2015.

40. Biliran H Jr, Wang Y, Banerjee S, Xu H, Heng H, Thakur A, Bollig A, Sarkar FH and Liao JD: Overexpression of cyclin D1 promotes tumor cell growth and confers resistance to cisplatin-mediated apoptosis in an elastase-myc transgene-expressing pancreatic tumor cell line. Clin Cancer Res 11: 6075-6086, 2005.

41. Aggarwal P, Vaites LP, Kim JK, Mellert H, Gurung B, Nakagawa H, Herlyn M, Hua X, Rustgi AK, McMahon SB and Diehl JA: Nuclear cyclin D1/CDK4 kinase regulates CUL4 expression and triggers neoplastic growth via activation of the PRMT5 methyltransferase. Cancer Cell 18: 329-340, 2010. 
42. Shields BJ, Hauser C, Bukczynska PE, Court NW and Tiganis T: DNA replication stalling attenuates tyrosine kinase signaling to suppress S phase progression. Cancer Cell 14: 166-179, 2008.

43. Jirawatnotai S, Hu Y, Michowski W, Elias JE, Becks L, Bienvenu F, Zagozdzon A, Goswami T, Wang YE, Clark AB, et al: A function for cyclin D1 in DNA repair uncovered by protein interactome analyses in human cancers. Nature 474: 230-234, 2011.

44. Yao Z, Fenoglio S, Gao DC, Camiolo M, Stiles B, Lindsted T, Schlederer M, Johns C, Altorki N, Mittal V, et al: TGF-beta IL-6 axis mediates selective and adaptive mechanisms of resistance to molecular targeted therapy in lung cancer. Proc Natl Acad Sci USA 107: 15535-15540, 2010 .

45. Wang S, Long S, Xiao S, Wu W and Hann SS: Decoction of chinese herbal medicine fuzheng kang-ai induces lung cancer cell apoptosis via STAT3/Bcl-2/caspase-3 pathway. Evid Based Complement Alternat Med 2018: 8567905, 2018.

46. Yu H and Jove R: The stats of cancer-new molecular targets come of age. Nat Rev Cancer 4: 97-105, 2004

47. Dechow TN, Pedranzini L, Leitch A, Leslie K, Gerald WL, Linkov I and Bromberg JF: Requirement of matrix metalloproteinase-9 for the transformation of human mammary epithelial cells by Stat3-C. Proc Natl Acad Sci USA 101: 10602-10607, 2004.

48. El-Badrawy MK, Yousef AM, Shaalan D and Elsamanoudy AZ: Matrix metalloproteinase-9 expression in lung cancer patients and its relation to serum mmp-9 activity, pathologic type, and prognosis. J Bronchology Interv Pulmonol 21: 327-334, 2014.
49. Backstrom JR and Tökés ZA: The 84-kDa form of human matrix metalloproteinase-9 degrades substance $P$ and gelatin. J Neurochem 64: 1312-1318, 1995.

50. Ao N, Liu Y, Bian X, Feng H and Liu Y: Ubiquitin-specific peptidase 22 inhibits colon cancer cell invasion by suppressing the signal transducer and activator of transcription 3/matrix metalloproteinase 9 pathway. Mol Med Rep 12: 2107-2113, 2015.

51. Kim JH and Park B: Triptolide blocks the STAT3 signaling pathway through induction of protein tyrosine phosphatase SHP-1 in multiple myeloma cells. Int J Mol Med 40: 1566-1572, 2017.

52. Niu F, Li Y, Lai FF, Ni L, Ji M, Jin J, Yang HZ, Wang C, Zhang DM and Chen XG: LB-1 exerts antitumor activity in pancreatic cancer by inhibiting HIF- $1 \alpha$ and Stat 3 signaling. J Cell Physiol 230: 2212-2223, 2015.

53. Kim SM, Kwon OJ, Hong YK, Kim JH, Solca F, Ha SJ, Soo RA, Christensen JG, Lee JH and Cho BC: Activation of IL-6R/JAK1/STAT3 signaling induces de novo resistance to irreversible EGFR inhibitors in non-small cell lung cancer with T790M resistance mutation. Mol Cancer Ther 11: 2254-2264, 2012.

54. Maji S, Shriwas O, Samal SK, Priyadarshini M, Rath R, Panda S, Das Majumdar SK, Muduly DK and Dash R: STAT3- and GSK3 $\beta$-mediated Mcl-1 regulation modulates TPF resistance in oral squamous cell carcinoma. Carcinogenesis 40: 173-183, 2019. 